\title{
Mathematical Modeling of Fluid Flow and Heat Transfer in Petroleum Industries and Geothermal Applications 2020
}

\author{
Mehrdad Massoudi
}

Citation: Massoudi, M. Mathematical Modeling of Fluid Flow and Heat Transfer in Petroleum Industries and Geothermal Applications 2020. Energies 2021, 14 5104. https://doi.org/10.3390/ en14165104

Received: 16 August 2021

Accepted: 17 August 2021

Published: 19 August 2021

Publisher's Note: MDPI stays neutral with regard to jurisdictional claims in published maps and institutional affiliations.

Copyright: (c) 2021 by the author. Licensee MDPI, Basel, Switzerland. This article is an open access article distributed under the terms and conditions of the Creative Commons Attribution (CC BY) license (https:// creativecommons.org/licenses/by/ $4.0 /)$.
U.S. Department of Energy, National Energy Technology Laboratory (NETL), Pittsburgh, PA 15236-0940, USA; Mehrdad.Massoudi@NETL.DOE.GOV

\begin{abstract}
In this Special Issue, all aspects of fluid flow and heat transfer in geothermal applications, including the ground heat exchanger, conduction, and convection in porous media, are considered. The emphasis here is on mathematical and computational aspects of fluid flow in conventional and unconventional reservoirs, geothermal engineering, fluid flow and heat transfer in drilling engineering, and enhanced oil recovery (hydraulic fracturing, steam-assisted gravity drainage (SAGD), $\mathrm{CO}_{2}$ injection, etc.) applications.
\end{abstract}

Keywords: mathematical modeling; computational fluid dynamics (CFD); drilling; porous media; multiphase flow; hydraulic fracturing; geothermal; enhanced oil recovery

\section{Introduction}

This Special Issue is a continuation of the previous Special Issue, "Mathematical Modeling of Fluid Flow and Heat Transfer in Petroleum Industries and Geothermal Applications", published in 2019. Geothermal energy is the thermal energy generated and stored in the Earth's core, mantle, and crust. Geothermal technologies are used to generate electricity and heat and cool buildings. To develop accurate models for heat and mass transfer applications involving fluid flow in geothermal applications or reservoir engineering and petroleum industries, a basic knowledge of the rheological and the transport properties of the materials involved (for example, drilling fluid, rock properties, etc.), especially in high-temperature and high-pressure environments, are needed. In this Special Issue, all aspects of fluid flow and heat transfer in geothermal applications, including the ground heat exchanger, conduction, and convection in porous media, are considered. The emphasis here is on mathematical and computational aspects of fluid flow in conventional and unconventional reservoirs, geothermal engineering, fluid flow and heat transfer in drilling engineering, and enhanced oil recovery (hydraulic fracturing, steam-assisted gravity drainage (SAGD), $\mathrm{CO}_{2}$ injection, etc.) applications. I would like to thank all the authors who contributed to this Special Issue. A brief outline of each paper is given below.

One of the major problems in petroleum industries is related to the agglomeration of gas bubbles inside electrical submersible pumps (ESP) impellers; this can cause lower pump performance and degradation, resulting in mild to severe deterioration (surging/gas pockets). Ali et al. [1] compare the existing empirical two-phase models with their singlestage centrifugal pump results to see their validity and working range. By performing a CFD-based (computational fluid dynamics) numerical analysis, they also discuss gashandling design methods, which are helpful in enhancing the multiphase performance of ESPs.

In many cases involving fluid flows in geothermal applications and petroleum industries, pipe flow is probably the most studied problem. Alves dos Santos Júnior et al. [2] look at the fully developed laminar flow of a fluid through a corrugated cylindrical duct using the Galerkin-based integral method. They consider a heavy oil with a relative density of $0.9648\left(14.6^{\circ} \mathrm{API}\right)$ and temperature-dependent viscosities ranging from 1715 to $13,000 \mathrm{cP}$. 
Their results are given for different fluid parameters, such as the Fanning friction factor, Reynolds number, shear stress, and pressure gradient.

An important problem in the oil industry is the disposal of produced water, and membrane-separation technology appears to be an essential tool in the treatment of these waters. Magalhães et al. [3] develop a mathematical model for simulating the oil/water separation by using a ceramic membrane, along with the CFD code, Ansys CFX ${ }^{\circledR} 15$. They present the results for oil concentration, pressure, and velocity distributions, and permeation velocity. Their results indicate that the temperature strongly influences the flow behavior.

To maintain reservoir pressure and improve the oil sweep efficiency, waterflooding is commonly used. However, injecting a cold liquid can cause stresses which can lead to fractures. These thermally induced fractures (TIFs) can cause non-uniform distribution of fluid flow in wellbores, reducing the sweep efficiency and initiating the early water breakthrough in the production wells nearby. Almarri [4] uses a 3D reservoir simulator coupled with 2D finite element TIF with geomechanical models to match an injector (NI6) in the N Field sector reservoir model. The author improves the reservoir and the geomechanical models by validating them via the observed TIF onset and propagation periods.

Among the many different flow simulation models, the embedded discrete fracture model (EDFM) is known to provide a reasonable balance between efficiency and accuracy. Shao et al. [5] propose an integrally embedded discrete fracture model (iEDFM) for anisotropic formations; they use a structured mesh and fracture element, the latter consisting of a group of connected fractures embedded in the matrix grid. They derive an analytic pressure distribution for the point source expressed in terms of the permeability tensor. They also consider a two-phase flow problem, where they analyze the effect of the matrix anisotropy on the simulation result.

Szott et al. [6] develop a model for an ongoing project (24 years) for acid gas sequestration in a deep geological structure. The model is based on the available operational and monitoring data, and it can help determine the basic characteristics of the sequestration process. Their simulation results can predict the capacity of the analyzed structure by finding the optimum situation for increased sequestration. The importance of fluids saturation and compositions, along with the time for fluid migrations and the gas transition, are also discussed.

In many geothermal reservoirs, there are large fracture apertures and, as a result, severe loss of circulations can occur; this is very costly and may lead to stuck pipe, well instability, and well abandonment. One of the techniques used to overcome this problem is by adding lost-circulation materials (LCMs) to seal the fractures. Lee and Dahi Taleghani [7] perform parametric studies in order to better understand the various thermal effects on the sealing mechanisms of LCMs. They use the computational fluid dynamics (CFDs) approach along with the discrete element method (DEM) to accurately describe the physics of sealing by granular materials. They study the fracture-sealing capability by considering the effects of four parameters, including fluid viscosity, particle size, friction coefficient, and Young's modulus. They notice that thermally degraded properties can lead to inefficient fracture sealing.

Amongst the various modeling approaches to study the horizontal oil-water flow is the Algebraic Interfacial Area Density (AIAD) model. Höhne et al. [8] look at this model and compare the standard ANSYS Fluent Eulerian Interface Capabilities (namely, MultiFluid VOF) without AIAD, and ANSYS CFX with AIAD implemented via user functions. Their numerical results, compared with the existing experimental data, indicate that the accuracy of the horizontal oil-water flow can be increased when using the regime transition capabilities such as those offered by the AIAD model.

A conventional method in secondary oil recovery is to inject water into the oilsaturated porous rock. Gołabek et al. [9] discuss the use of scaling and dimensional analysis and assess the viability of conventional modeling of immiscible displacement. They use the Buckingham $\Pi$ theorem to generate all possible combinations of dimen- 
sionless products for physical variables describing the phenomenon. They analyze the laboratory tests and statistically verify the quantities describing both the dependent and the independent experimental variables.

Apparent gas permeability (AGP) is an important parameter that needs to be considered in most reservoir simulations. Li et al. [10] develop an AGP model, coupling multiple mechanisms, to study gas transport in a multiscale shale matrix. Various effects, such as deformation of the porous media, irreducible water saturation, and gas ab-/de-sorption are considered. They also look at the effective pore size distribution (PSD) and AGP under different water saturation. Their results indicate that the presence of irreducible water leads to a decrease of AGP, and at low water saturation conditions, irreducible water tends to occupy some of the pores.

Sukharev [11] looks at the flow of a multicomponent fluid through a pipeline system of arbitrary configuration. The author introduces a criterion similar to entropy in thermodynamic processes, characterizing the degree of closeness of a multicomponent mixture to an equilibrium state. If there are no phase transitions, the main problem reduces to determining the composition of the fluid for each pipeline based on the values of the concentration of the components. By providing a numerical example where the distribution of a three-component mixture is given, the author shows the feasibility of the proposed computational procedure.

Many suspensions, composed of fluid and solid particles, behave as non-Newtonian fluids with rheological characteristics such as yield stress, thixotropy, and shear-thinning/thickening, etc. Li et al. [12] look at the flow of a non-linear fluid with yield stress exhibiting shear-thinning effects in the following geometries: (i) a straight channel, (ii) a channel with a crevice, and (iii) a pipe with a contraction. They assume that the suspension behaves as a Herschel-Bulkley fluid. By numerically solving these problems, they notice that for flows with low Reynolds number and high Bingham number, an unyielded plug may form in the center of the channel.

$\mathrm{Wu}$ and $\mathrm{Wu}$ [13] study a fluid-particle suspension by modeling the mixture as a non-linear single-phase fluid where the migration of the solid particles is incorporated by using a particle flux equation, where the effects of particle migration on the momentum and internal energy of the suspension are considered. They consider two problems: (i) flow in a sudden expansion channel and (ii) flow between two rotating cylinders. They notice that the particle flux model converges without any problems; they use the PISO-SIMPLE transient solver.

In measuring the yield stress of gelled waxy crude oil, the inertia of the measuring device can cause the actual shear rate to be different from the setting one, and it may become necessary to modify the shear rate history in model validation. Sun et al. [14] use a fractal model for the structural description of the waxy gel and Marrucci's model for the time evolution of the stress where elastic and viscous effects are included. It is shown that Marrucci's model provides much better results compared to the Maxwell model, as it incorporates the non-linear viscoelastic effects of the waxy crude gels in the stress evolution equation.

Funding: This research received no external funding.

Acknowledgments: Without the help of qualified reviewers, it would not have been possible to organize this Special Issue. I am grateful to all the anonymous reviewers for their help. A personal note of appreciation and gratitude to Howland (Hao) Wu and the editorial staff at Energies; without their help and assistance, Energies could not publish high-quality papers in a short period of time.

Conflicts of Interest: The author declares no conflict of interest. 


\section{References}

1. Ali, A.; Yuan, J.; Deng, F.; Wang, B.; Liu, L.; Si, Q.; Buttar, N.A. Research Progress and Prospects of Multi-Stage Centrifugal Pump Capability for Handling Gas-Liquid Multiphase Flow: Comparison and Empirical Model Validation. Energies 2021, $14,896$. [CrossRef]

2. Alves dos Santos Júnior, V.; Rodrigues de Farias Neto, S.; Gilson Barbosa de Lima, A.; Fernandes Gomes, I.; Buriti Galvão, I.; Maria Rufino Franco, C.; Evangelista Franco do Carmo, J. Heavy Oil Laminar Flow in Corrugated Ducts: A Numerical Study Using the Galerkin-Based Integral Method. Energies 2020, 13, 1363. [CrossRef]

3. Magalhães, H.L.F.; Moreira, G.; Gomez, R.S.; Porto, T.R.N.; Correia, B.R.B.; Silva, A.M.V.; Farias Neto, S.R.; Lima, A.G.B. Non-Isothermal Treatment of Oily Waters Using Ceramic Membrane: A Numerical Investigation. Energies 2020, $13,2092$. [CrossRef]

4. Almarri, M. Efficient History Matching of Thermally Induced Fractures Using Coupled Geomechanics and Reservoir Simulation. Energies 2020, 13, 3001. [CrossRef]

5. Shao, R.; Di, Y.; Wu, D.; Wu, Y.-S. An Integrally Embedded Discrete Fracture Model for Flow Simulation in Anisotropic Formations. Energies 2020, 13, 3070. [CrossRef]

6. Szott, W.; Łętkowski, P.; Gołąbek, A.; Miłek, K. Modelling of the Long-Term Acid Gas Sequestration and Its Prediction: A Unique Case Study. Energies 2020, 13, 4701. [CrossRef]

7. Lee, L.; Dahi Taleghani, A. Simulating Fracture Sealing by Granular LCM Particles in Geothermal Drilling. Energies 2020, 13, 4878. [CrossRef]

8. Höhne, T.; Rayya, A.; Montoya, G. Numerical Modelling of Horizontal Oil-Water Pipe Flow. Energies 2020, 13, 5042. [CrossRef]

9. Gołabek, A.; Szott, W.; Łẹtkowski, P.; Stopa, J. Similitude Analysis of Experiment and Modelling of Immiscible Displacement Effects with Scaling and Dimensional Approach. Energies 2020, 13, 5224. [CrossRef]

10. Li, X.; Liu, S.; Li, J.; Tan, X.; Li, Y.; Wu, F. Apparent Permeability Model for Gas Transport in Multiscale Shale Matrix Coupling Multiple Mechanisms. Energies 2020, 13, 6323. [CrossRef]

11. Sukharev, M. Fluid Mixing Nonequilibrium Processes in Industrial Piping Flows. Energies 2020, 13, 6364. [CrossRef]

12. Li, M.-G.; Feng, F.; Wu, W.-T.; Massoudi, M. Numerical Simulations of the Flow of a Dense Suspension Exhibiting Yield-Stress and Shear-Thinning Effects. Energies 2020, 13, 6635. [CrossRef]

13. Wu, Y.; Wu, W.-T. Modeling on Effect of Particle Sediment on Fluid Flow and Heat Transfer of Solid-Fluid Suspension. Energies 2021, 14, 487. [CrossRef]

14. Sun, M.; Jou, D.; Wang, Z. A Nonlinear Viscoelastic Model for the Yielding of Gelled Waxy Crude Oil. Energies 2021, 14, 536. [CrossRef] 